\title{
ANÁLISIS DE LA REPERCUSIÓN DEL CURSO ESPECIAL DE POSGRADO EN “GESTIÓN LOCAL DE SALUD”, EN LA ATENCIÓN A USUARIOS DE EBAIS DEL SECTOR TRES DE DESAMPARADOS
}

Teresita Flores Jiménez

\author{
Recibido 12-II-2003 • Aceptado 10-VI- 2003
}

\begin{abstract}
Resumen: A través del tiempo el CENDEISSS ha asumido un papel protagonista en la formación y capacitación del recurso humano de la Caja Costarricense de Seguro Social en aras de una mejor atención integral de la salud de los costarricenses. Como parte de esta misión, promueve y ejecuta el proceso de capacitación del curso especial de posgrado en "Gestión local de salud", orientado, hacia la transformación e implementación de un modelo readecuado de la atención de la salud que fortalece la atención con un enfoque biopsicosocial, acorde con las políticas de modernización, en el contexto de la Reforma del Sector Salud. En 1997, como parte del cumplimiento de su misión, el CENDEISSS da inicio al primer curso especial de posgrado en "Gestión Local de Salud", para médicos generales de los Equipos Básicos de Atención Integral en Salud (EBAIS) Posteriormente lo amplía para todos los profesionales del área de salud.

Dada la importancia de la capacitación para el personal de salud en el ámbito institucional, el presente estudio pretende, por lo tanto, enfatizar la función del CENDEISSS con respecto a la capacitación del personal del sector salud, y los cambios en la atención a los usuarios, producto de la capacitación recibida.
\end{abstract}

Palabras clave: Capacitación, Salud, Atención Básica, Aplicabilidad, Percepción, Usuario.

\section{Introducción}

\section{El problema y su importancia}

El Centro de Desarrollo Estratégico e Información en Salud y Seguridad Social (CENDEISSS), fue creado en 1974 como respuesta a la creciente necesidad de la especialización de profesionales en ciencias médicas y formación de técnicos paramédicos; consecuencia de la universalización de los Seguros Sociales en 1970 y la creación de la Facultad de Medicina de la Universidad de Costa Rica en 1964. El CENDEISSS se convirtió en protagonista de la formación y capacitación de los recursos humanos en salud, para dar respuesta adecuada a la demanda de servicios de la Caja Costarricense de Seguro Social. (C.C.S.S).

Las funciones de ésta Institución tienen su origen en el cambio que, a partir de la Reforma al Sector Salud a finales de los 80 , se produce en el modelo nacional de prestación de servicios de salud. Este proceso se propone fortalecer la capacidad de dirección y planeación en dicho servicio, mejorando la cobertura, la accesibilidad, oportunidad, calidad y ámbito de operación de estos servicios, de acuerdo con las necesidades de la población en función de 
las condiciones económicas del país, se divide así el territorio nacional en siete regiones geográficas:

\section{- Región Huetar Norte \\ - Región Huetar Atlántico \\ - Región Brunca \\ - Región Pacífico Central \\ - Región Central Norte \\ - Región Central Sur \\ - Región Chorotega}

Cada región, a su vez, se divide en áreas de salud, conformadas así mismo por sectores. Cada área abarca un cantón con una población entre 15000 y 40000 personas en zonas rurales y en zonas urbanas de 30000 a 60000 habitantes. Los sectores incluyen de 4000 a 4500 habitantes y están atendidos por un Equipo Básico de Atención Integral en Salud (EBAIS).

El EBAIS es conceptualizado por la C.C.S.S. como un equipo básico formado por un médico general, una auxiliar de enfermería y un asistente de atención primaria en salud (ATAPS); con el apoyo de profesionales en trabajo social, farmacia, odontología, enfermería, psicología, nutrición, medicina familiar y comunitaria.

Por otra parte, al firmarse en 1996 la Reforma del Sector de Salud, se implementa un modelo readecuado de atención integral con la participación social de las comunidades. Como parte de esta reforma, la Escuela de Salud Pública de la Universidad de Costa Rica (UCR) se compromete con la C.C.S.S. a fortalecer las destrezas y conocimientos de los profesionales en salud, modificando la concepción de la enseñanza biologista, concentrada en servicios hospitalarios, por una acción centrada y orientada en la proyección hacia la comunidad. De esta forma, se le da mayor participación a los actores externos en la toma de decisiones para la detección de problemas de salud que les afectan y la búsqueda de soluciones en equipo. Lo importante de esta nueva visión es que la comunidad tiene una función activa en todo el proceso, al participar en la solución de los problemas con la guía y orientación del personal de salud a escala local. La atención no se enfoca únicamente en la enfermedad, sino que va más allá para encontrar alternativas de solución a las causas de esta.

En este marco, la función del CENDEISSS, en coordinación con la UCR y en especial con la Escuela de Salud Pública, es brindar una educación continua y permanente al profesional en ciencias de la salud; no solo ver al estudiante como objeto sino como sujeto de la educación y de su propio aprendizaje. Para lograr esta misión, se organizan núcleos de capacitación en cada región del país, dirigido a estos profesionales que laboran en el primer nivel de atención. Uno de los cursos de capacitación que contiene los elementos mencionados de la Reforma del sector salud, en lo referente al modelo readecuado de atención integral es el curso especial de posgrado en "Gestión local de salud" el cual es de interés para la presente investigación.

Este curso se imparte con una extensión de diez meses, dio inicio en 1996 y se ofrece regularmente todos los años, desde entonces (CENDEISSS, 2002).

El proceso de capacitación se compone de once módulos, los cuales se mencionan a continuación:

1. El sistema nacional de salud y su proceso de modernización.

2. Planificación estratégica de los servicios de salud.

3. Participación social en el campo de la salud.

4. Elementos de salud familiar.

5. Sistemas de información social en salud en el primer nivel de atención.

6. Análisis de situación integral de salud. 
7. Fase instrumental para la planificación estratégica de los servicios de salud.

8. Vigilancia epidemiológica y calidad de los servicios.

9. Ambiente y salud.

10. Introducción al estudio de la calidad de los servicios de salud.

11. Análisis de la gestión clínica para el primer nivel de la atención.

El CENDEISSS, tiene la responsabilidad de regular y ser líder en el desarrollo del recurso humano Institucional. Para la realización de las actividades educativas requeridas, el CENDEISSS cuenta con diferentes procesos, uno de ellos es el de avalar estas actividades por lo que se aprobó el curso especial de posgrado en "Gestión local de salud" para las siete regiones del país. Este curso responde a las necesidades planteadas por las comunidades costarricenses para mejorar la calidad de los servicios de salud.

Otros procesos con que cuenta el CENDEISSS para realizar sus funciones, son la supervisión, el registro y el control de actividades educativas, mediante las cuales se brinda a funcionarios de la Caja Costarricense de Seguro Social la asesoría para la detección de las necesidades de capacitación en los servicios, como también la planificación de diseños curriculares y proyectos educativos; las estrategias metodológicas y los sistemas de evaluación del aprendizaje: antes, durante y después del proceso.

A pesar de la importante labor que realiza el CENDEISSS, la evaluación en general y específica de los resultados del aprendizaje en las diferentes acciones e instancias, a escala local, es escasa. Actualmente se cuenta con un estudio sobre evaluación de resultados del curso especial de posgrado en "Gestión local de salud" de la dirección Central Norte (Mora A., 1999). Sin embargo, este estudio no toma en cuenta la población beneficiaria, la cual es el objetivo más importante dentro de las políticas de salud de la C.C.S.S.

El presente estudio pretende, por lo tanto, enfatizar en la función del CENDEISSS con respecto a la capacitación del personal del sector salud, identificar los posibles cambios en la atención a los usuarios, producto de la formación recibida en Gestión local de Salud. Se persiguen en esta investigación cuatro aspectos: 1. Identificar en la propuesta curricular los elementos relacionados con la atención al usuario. 2 . La aplicabilidad del conocimiento en gestión local de los profesionales en salud que integran los EBAIS seleccionados de la comunidad de Desamparados que fueron capacitados en gestión local y que recibieron la capacitación. 3. Además se pretende determinar la percepción del usuario en relación con la calidad de los servicios. 4. Por último establecer la relación entre la formación recibida en el curso "especial de posgrado en "Gestión local de salud" y la atención brindada al usuario.

\section{Planteamiento del problema}

¿Cuáles son las repercusiones del proceso de formación en el curso especial de posgrado en "Gestión local de salud" en relación con la atención que se brinda al usuario del Sector Tres de Desamparados?

De acuerdo con el planteamiento del problema descrito, la investigación plantea los siguientes objetivos:

\section{Objetivo general}

Evidenciar la aplicación de conocimientos del curso especial de posgrado en "Gestión local de salud", en la atención a 
los usuarios de los servicios de salud de los EBAIS: Guido 3, Fátima, y Guatuso del Sector Tres de Desamparados.

\section{Objetivos específicos}

- Identificar en la propuesta curricular del curso especial de posgrado en "Gestión local de salud", los elementos prácticos relacionados con la atención al usuario.

- Identificar la aplicabilidad de los elementos prácticos de capacitación en Gestión local de salud recibidos por los profesionales, en los servicios brindados a los usuarios de los EBAIS.

- Determinar la percepción del usuario en relación con la calidad de atención a nivel práctico de los servicios que recibe de los profesionales de salud que llevaron el curso.

- $\quad$ Establecer la relación entre la formación recibida en el curso especial de posgrado en "Gestión local de salud" y la atención brindada al usuario.

Para la realización de la presente investigación se organizó el marco teórico en dos apartados el primero llamado marco institucional en el cual se hace mención a la función del CENDEISSS y Equipos Básicos de Atención Integral (EBAIS). En el segundo apartado se conceptualizan los elementos relacionados a la Reforma del Sector Salud.

\section{Marco Institucional}

\section{El CENDEISSS}

Estructuralmente el CENDEISSS es una dependencia de la Gerencia Médica de la Caja Costarricense de Seguro Social, funcionalmente conduce y regula el desarrollo del recurso humano institucional, siendo la capacitación su labor sustancial como ya se indicó anteriormente.

\section{Equipos Básicos de Atención Integral (EBAIS)}

Para la readecuación del modelo de atención de la salud, la Caja Costarricense de Seguro Social plantea, como principal estrategia, el modelo de atención en el nivel local mediante la asignación de un Equipo Básico de Atención Integral. El EBAIS es conceptualizado por la C.C.S.S. como un equipo integrado por un médico general, una auxiliar de enfermería y un asistente de atención primaria (ATAP). Además un equipo de apoyo integrado por profesionales en: trabajo social, farmacia, enfermería, odontología, psicología, nutrición, medicina familiar y comunitaria; todos juntos conforman el equipo de salud.

El Ministerio de Salud señala la misión del EBAIS como la instancia responsable de la atención integral de la salud de las personas en un espacio poblacional, donde cada unidad contiene la atención de los procesos determinantes de los problemas de salud-enfermedad (C.C.S.S, 1993).

En constante interacción los equipos de apoyo y los EBAIS deben realizar un trabajo coordinado y sistemático, lo cual implica un amplio conocimiento actualizado del sector y del área total. Dentro de la responsabilidad de estos equipos está la elaboración de planes locales de trabajo, el permanente control de las funciones y actividades realizadas, la evaluación de los resultados obtenidos, la valoración de la calidad de los servicios brindados el trabajo en equipo, y la ejecución coordinada de las acciones cotidianas.

El Ministerio de Salud y la C.C.S.S. definen seis ambientes de desempeño de los EBAIS que son: el familiar, la comunidad, los establecimientos públicos y privados, el educativo, el laboral y el establecimiento de salud. Como se puede observar 
las funciones definidas anteriormente requieren amplios conocimientos y la aplicación de destrezas; así como la transformación de la actitud de los ejecutores que son el personal de salud y la comunidad local. Para el cumplimento de estas nuevas funciones es de vital importancia capacitar el personal de salud, quien a su vez educará a la comunidad y generará la participación comunal en la promoción de la salud.

El curso especial de posgrado en "Gestión local de salud" se propone cumplir con esta responsabilidad como parte de la capacitación que deben tener todos los miembros de salud que laboren en el ámbito local.

\section{Marco conceptual}

La Reforma del Sector Salud en Costa Rica

Con el fin de aumentar la eficiencia en la administración de los recursos, las autoridades de salud han formulado el proyecto de Reforma del Sector Salud que tiene su origen en la ley 74141 de 1993 implementado bajo el nombre de Modernización del Sistema de Salud. Dicha reforma implica diversos cambios entre ellos busca corregir y mejorar la capacidad operativa de las instituciones del sector salud e introducir modificaciones profundas al marco de salud por medio de los Compromisos de Gestión y el componente de rectoría del Ministerio de Salud. Como primer elemento los compromisos de gestión permiten rendición de cuentas, organización interna, adecuado manejo de la información y ordenamiento presupuestario; cada institución debe crear una cultura de compromiso de gestión en sus funcionarios para mejorar la participación del personal en los distintos procesos. El segundo elemento consiste en asignar al Ministerio de Salud la dirección, coordinación, conducción, vigilancia, planificación de la salud, regulación, control, evaluación, investigación y desarrollo tecnológico. La función rectora del Ministerio de Salud y los compromisos de gestión permiten asegurar el mejoramiento de los servicios de salud, contribuir y garantizar los principios fundamentales de universalidad, solidaridad en el financiamiento e integridad en la prestación de servicios de salud a las personas y equidad en el acceso a los mismos.

Estos principios se conceptualizan de la siguiente manera: (C.C.S.S., 1997).

Universalidad: El sistema nacional de salud deberá cubrir o proteger a todos los ciudadanos con servicios de atención a las personas y al ambiente de manera integral, incorporándolos al sistema con plenos derechos y deberes y sin limitaciones geográficas, sociales, económicas o culturales.

Solidaridad: El nuevo modelo será altamente solidario, lo que se entiende como el medio por el cual cada individuo cotiza en el Seguro Social de acuerdo con sus posibilidades, para un fondo común en el cual el que más cotiza permite que los servicios puedan ser brindados a la población que no los puede financiar y que en su mayoría, es la que más requiere de atención directa. Para viabilizar este principio, es necesario decretar la obligatoriedad de los seguros sociales.

Equidad: Se entiende como la distribución justa de los recursos del Sector. Se encuentra íntimamente relacionada con el criterio de accesibilidad de cada población a los servicios sin barreras sociales, políticas o económicas. En el modelo readecuado, este principio deberá hacerse efectivo alcanzando dos grandes metas: Regímenes de Enfermedad y Maternidad y Riesgos del Trabajo.

Concepto de salud

Con el nuevo modelo de la atención de la salud se conceptualiza ésta como un estado dinámico susceptible de ser alcanzado mediante aproximaciones sucesivas en donde se reconocen los vínculos entre el bienestar de las personas, sus estilos de 
vida y el equilibrio con los factores ambientales. (Aguilar, 2000).

Esta definición de salud conlleva entender por modelo de atención una caracterización teórica del sistema de servicios de salud del país con base en sus principales elementos políticos, filosóficos, éticos, culturales, estructurales organizativos y funcionales; en especial en la actualización de normas y su aplicación por la vida con control y evaluación en áreas de importancia estratégica como la calidad de la salud, economía, financiamiento de la salud y el desarrollo tecnológico, entre otros.

\section{El modelo de la atención biopsicosocial}

Este cambio de modelo de salud, implica que el sujeto de la atención dejará de ser exclusivamente el individuo, para dirigirla principalmente a la familia, a la comunidad y al ambiente.

El modelo readecuado de atención propiciará un cambio de actitud en la sociedad, con respecto a la salud, pues ésta dejará de ser un problema eminentemente individual para convertirse en una situación colectiva, siendo responsabilidad de todos el mantenerla, preservarla y mejorarla. El nuevo modelo de la salud implica cambios importantes en tres áreas, a saber: en el auto cuidado de la salud, en la ejecución de actividades y en la gestión de los servicios. En este contexto promover e impulsar la participación social será competencia de cada centro de salud, llámese EBAIS, clínica, hospital y todas las instancias que se encarguen de la capacitación y formación de personal de salud.

Curso especial de posgrado en

"Gestión local de salud"

En 1997 como parte del cumplimiento de su misión el CENDEISSS da inicio al primer curso especial de posgrado en "Gestión local de salud", para médicos generales de los Equipos Básicos de Atención integral en Salud (EBAIS). Este curso es un esfuerzo conjunto de la C.C.S.S. por intermedio del CENDEISSS, y la Universidad de Costa Rica (UCR) específicamente la Escuela de Salud Pública. Se constituye así una alianza estratégica entre la institución que se encarga de la prestación de los servicios de salud en el país, y la Universidad que históricamente ha acompañado el desarrollo de la salud pública costarricense.

A partir del segundo curso, impartido en 1999 se agrega una nueva variable que es la heterogeneidad de disciplinas. El curso ya no se dirige únicamente a médicos generales de los EBAIS, sino que incluye una población interdisciplinaria donde participan diversos profesionales en salud como: farmacéuticos, médicos, trabajadores sociales, psicólogos, enfermeras y microbiólogos, los cuales forman parte del equipo de apoyo de los EBAIS.

El proceso de capacitación se planifica mediante la utilización de una metodología a distancia y sesiones presenciales de ocho horas cada quince días, aplicando tareas específicas e investigaciones sobre el tema en estudio. Asimismo, se utilizan técnicas como: talleres, análisis de problemas, estudio de casos, estudios dirigidos; discusión de lecturas asignadas, análisis integrales e interdisciplinarios de casos clínicos, de relaciones familiares y de problemas de salud del área, diseño de un proyecto y planes de trabajo. Los módulos de capacitación fueron elaborados por equipos interdisciplinarios de profesionales, provenientes de la Caja Costarricense de Seguro Social, del Ministerio de Salud y de las Universidades Estatales, mediante el modelo para la elaboración de módulos de autoaprendizaje llamado Modelo de Unidad Sistémica Estructurada MOUSE (C.C.S.S., 1999).

Para la realización del presente estudio se escogieron los módulos 3, 4, 6, 10 y 11 , los cuales contienen los elementos de formación necesarios para que los profesionales que laboran en los EBAIS desarrollen 
conocimientos teóricos-prácticos que orienten su labor diaria en beneficio de los usuarios. A continuación se resume el contenido de cada uno de los módulos seleccionados.

El módulo 3 (Participación social en el campo de la salud) es bastante explícito, en cuanto a propiciar el acceso de la población a los servicios de salud más humanos y de calidad; elementos básicos, para lograr la participación social en el campo de la salud. El contenido está centrado en la participación comunitaria en salud, en donde se destaca la planificación participativa y los elementos operativos como: promoción, organización e identificación de actores sociales. Es evidente, según el contenido del módulo, que los profesionales de la salud utilizan algunas estrategias que los llevan a insertarse dentro de la dinámica de la comunidad, para identificar los recursos con que cuentan y las necesidades o problemas. Entre ellas: visitas a personas o grupos, participar en actividades que se realizan en la comunidad y en los centros educativos; en este módulo el Análisis de la Situación de Salud integral (ASIS) es señalado como estrategia básica para lograr lo anterior. Estas estrategias están muy relacionadas con la aplicabilidad de los objetivos específicos por lo tanto es un módulo fundamental para el conocimiento de la comunidad e impulsar la participación social en la salud.

En el módulo 4 (Elementos de salud familiar) el propósito es desarrollar estrategias para el logro de la atención integral. Recalca la importancia de la salud familiar. Esta intención se establece en el propósito, los objetivos y el contenido, en el cual se menciona que el profesional de salud debe abordar al usuario como un ser integral en el estudio de casos, e identificar en la práctica al EBAIS como un equipo interdisciplinario, organizando los servicios para la atención del usuario por grupos de edad y prioridad de la atención. Este módulo brinda herramientas básicas al profesional de la salud para el quehacer cotidiano de los servicios que ofrece al individuo, a la familia y a la comunidad.

El módulo 6 (Análisis de situación integral de salud) contiene información amplia sobre el análisis de la situación integral para contribuir con la calidad de salud de las comunidades. Durante el proceso de capacitación de los profesionales se proponía el desarrollo de un trabajo donde se evidencia la relación teórica-práctica al realizar estudio de casos.

En el módulo 10 (Introducción al estudio de la calidad de los servicios de salud) interesa que los profesionales identifiquen si la labor que realizan es de calidad y cuál es su marco de referencia para ello dentro de las estrategias propuestas para establecer la relación teórica-práctica, los profesionales deben darse a la tarea de identificar los elementos que caracteriza la calidad de atención en las diferentes actividades que se realizan en la solución de un caso de estudio. Este es un módulo básico para la labor del profesional de salud a nivel de los EBAIS, que permite desarrollar el ASIS en la comunidad con la colaboración del equipo de apoyo.

El módulo 11 (Análisis de la gestión clínica para el primer nivel de atención) se resalta la importancia de la gestión clínica para el primer nivel de atención y la relación del equipo de salud con el usuario.

El contenido se centra en el elemento clave de la reforma del sector salud; la cual va dirigida a que el usuario tenga una participación activa en las actividades que se realizan para el cuidado de la salud. Se mencionan como estrategias principales para la comunicación de profesional con usuario y la comunidad la visita domiciliaria, la referencia y la contrarreferencia. También se retoma el deber ser de la gestión clínica en relación con mejorar la calidad técnica y humana de los equipos de salud. 


\section{La atención primaria}

La atención primaria de salud es fundamentalmente asistencia sanitaria puesta al alcance de todos los individuos y familias de la comunidad, por medios que les sean aceptables, con su plena participación y a un costo que la comunidad y el país puedan soportar.

La "Gestión local de salud" está claramente ligada con la comunidad, la cual se debe hacer con un criterio de influencias interdependientes de carácter social, político y cultural. La Organización Panamericana de la Salud (O.P.S., 1990). considera la promoción, la prevención, la curación y la rehabilitación de la salud como un proceso de construcción colectiva y participativa donde los actores principales son las comunidades y los agentes de salud.

La calidad de la atención

Por ser la calidad de la atención un producto de múltiples interrelaciones, se propone una perspectiva en la que se incorpora el acceso a los recursos y su asignación a los diferentes segmentos de la población, de acuerdo con sus condiciones de vida. Los atributos de oportunidad, continuidad y coordinación, son también determinantes importantes de la calidad de la atención. En el estudio de la calidad de la atención de los servicios se profundiza en el análisis de estos, en relación con las necesidades de salud de la población adscrita y con la óptima organización y utilización de los recursos disponibles para responder a esas necesidades. Además se incorporan otros aspectos igualmente importantes como:

- $\quad$ La oportunidad de la atención, se refiere a que ésta no demora y pueda evitar posteriores complicaciones a quien la requiera.

- La satisfacción de los usuarios. Se determina por la calidad de atención que reciben de las personas que les brindan el servicio solicitado.

Por ello, los esfuerzos de los servicios de salud deben dirigirse a que la población comprenda y se apropie de los conocimientos, actitudes y prácticas que hagan posible su participación activa y crítica en los procesos de control de calidad. Es importante tomar en cuenta la satisfacción de los usuarios, porque proporciona información del éxito del proveedor para responder a esas necesidades. Esta es la mejor representación de ciertos componentes de la definición de calidad, especialmente en aquellos que concierne a las expectativas, valoraciones y necesidades sentidas y que deben reflejar los esfuerzos realizados en la educación y en la democratización de los conocimientos en salud por parte de los proveedores.

\section{Estrategia metodológica}

Tipo de investigación

La investigación que se realizó fue de tipo cualitativo, en la cual se produjeron datos descriptivos, para trascender hacia posiciones explicativas e interpretativas al transcribir las propias palabras de las personas, habladas o escritas, y las conductas observadas de los informantes de un modo natural (Hernández, 1991). Esto con el fin de realizar una comprensión detallada de sus perspectivas dentro de un marco contextual, observando a las personas en su labor cotidiana, en este caso a los funcionarios del equipo de salud y a los usuarios que reciben la atención de salud en los EBAIS.

Fases y etapas

El proceso de investigación se efectuó en tres fases: análisis documental, trabajo de campo y descripción de resultados. La primera de ella corresponde al análisis documental la cual se inició con una revisión 
bibliográfica de documentos relacionados con el tema y un análisis documental del programa y módulos seleccionados del curso especial de posgrado en "Gestión local de salud", con el objetivo de identificar, en la propuesta curricular, del curso citado, los elementos relacionados con la atención al usuario en el nivel local. Por último se elaboró una matriz para resumir la información obtenida del análisis de los módulos seleccionados para la presente investigación.

La segunda fase, consistió en trabajo de campo, conllevó tres etapas: Negociación de entrada, interacción con profesionales en salud participantes en el curso especial de posgrado en "Gestión local de salud", y consulta a usuarios de los servicios de salud.

En la etapa "negociación de entrada" se realizaron las coordinaciones con la jefatura de enfermería y médica del Sector Tres de Desamparados necesarias para el respectivo permiso.

Se realizó interacción con los profesionales de salud que laboran en los EBAIS y recibieron el curso especial de posgrado en "Gestión local de salud" con el objetivo de identificar la aplicabilidad de los elementos de formación recibidos en la atención que brindan a los usuarios. Para obtener la información requerida se utilizó como instrumento la entrevista semiestructurada.

La consulta a usuarios se realizó por medio de la técnica de grupos focales con las personas que recibieron la atención en los EBAIS seleccionados, por medio de los cuales se determinó la percepción con respecto a la calidad de atención que recibieron. El promedio de usuarios que se atiende por día en los EBAIS de Guatuso y Fátima es de 30 personas. Se seleccionó una muestra del $30 \%$ que correspondió a un total de 9 usuarios. La población que se atiende en el EBAIS de los Guidos 3 por día es de un promedio de 40 usuarios; la muestra que corresponde al $30 \%$ de los mismos, es de 12 usuarios.

Para la realización de los grupos focales de los EBAIS seleccionados se aprovecharon los tiempos de espera en las filas, en horas de la madrugada entre 430 am y $6 \mathrm{am}$, de los usuarios para obtener una ficha; la cual le daría el derecho a ser atendidos. Se consideró importante recoger la información de esta forma, debido a que en este tiempo de espera el grupo estaba reunido por un objetivo en común, no existían interferencias en la comunicación y todos tenían oportunidad de opinar. Se realizó también un grupo focal con miembros del comité de salud de los EBAIS de Fátima y de Guatuso, se consideró la importancia que tienen los comités para las comunidades, lo cual fue una experiencia muy provechosa.

La tercera fase consistió en la descripción y análisis de resultados para establecer la relación del conocimiento de los profesionales en salud recibido en el curso especial de posgrado en "Gestión local de salud" y la calidad de atención brindada a los usuarios. La realización de esta fase se llevo a cabo en cinco etapas: Transcripción y depuración de la información recopilada por los participantes, descripción y análisis de la información por categorías, triangulación, conclusiones y recomendaciones. La transcripción se realizó grabando y escuchando las entrevistas, releyendo y transcribiendo el contenido en su forma original. El tratamiento de descripción y análisis que se le dio a la información obtenida consistió en organizar, depurar y analizar el contenido para lo cual se agrupó la información por categorías según los objetivos específicos. Con base en los resultados obtenidos de las entrevistas estructuradas a los profesionales, de los grupos focales y del análisis de los documentos, se realizó la triangulación y las conclusiones a la vez se llegó a establecer y proponer algunas recomendaciones.

Participantes y fuentes de información

Los participantes en el estudio fueron los usuarios que asistieron a recibir atención de salud a los EBAIS, y los profesionales de 
salud que integran y apoyan éstos equipos. Para su selección se tomó en cuenta los siguientes criterios: El médico a cargo debía estar capacitado en Gestión Local y los EBAIS pertenecer al sector Tres de Desamparados, el cual es un cantón de la provincia de San José y por lo tanto parte de la Región Central Sur. Los EBAIS seleccionados fueron: Fátima, Guatuso y Guido 3. Se escogió este cantón por su alta concentración de población y por ser accesible para la investigadora, pues es el cantón de residencia.

Para la escogencia de los usuarios se tomó en cuenta la población que se atiende por día en las diferentes consultas, siendo diferente en cada EBAIS:

- $\quad$ Para el EBAIS de Guatuso y Fátima participaron diez usuarios, ya que por día se atiende una población promedio de 30 usuarios.

- $\quad$ Para el EBAIS de Guido 3, los participantes fueron 12 usuarios; la población que se atiende por día es de aproximadamente de 40 usuarios.

- Para el EBAIS Fátima la muestra fue de 10 usuarios, la población que se atiende es de 20 a 25 usuarios por día.

Entre los participantes se encontraron mujeres y hombres adultos.

Análisis de los resultados de la información obtenida

Para el análisis de resultados se llevó a cabo las siguientes acciones:

Revisión de la información recopilada con el propósito de obtener categorías para el análisis, Construcción de las categorías tomando como marco de referencia los objetivos específicos, el marco conceptual e institucional y la información recopilada de fuentes y participantes lo que permitió agrupar la información para el análisis posterior.

Las dos categorías empleadas fueron la aplicabilidad de conocimientos y percepción del usuario en relación con la atención brindada. La definición conceptual se presenta a continuación.

Aplicabilidad de los conocimientos

La aplicabilidad se refiere a la práctica cotidiana que realizan los profesionales de salud en los EBAIS relacionada con la capacitación recibida en el curso especial de posgrado en "Gestión local de salud", las estrategias didácticas utilizadas para su desarrollo y su relación con la documentación revisada.

Percepción del usuario en relación con la atención recibida

Se considera la percepción del usuario sobre el tipo de atención que se brinda en el EBAIS en relación con servicios, horario, trato, compromiso con la comunidad y participación de actores externos en la toma de decisiones para la detección de problemas de salud y su solución, se valora la calidad de atención en relación con los procesos de trabajo de los EBAIS. Esta opinión puede ser positiva, negativa o neutra. La percepción del usuario permite determinar si los servicios que recibe se dan en forma integral y con calidad.

Es importante indicar que durante la investigación surgieron datos, opiniones e información que no se ubicaron totalmente, aunque sí se mencionan en las categorías seleccionadas, pero que pueden considerarse como emergentes por ejemplo: La consulta fluctuante de extranjeros indocumentados sobre todo nicaragüenses que vienen y van de un EBAIS a otro, la poca motivación de algunos miembros de las comunidades investigadas para colaborar con la limpieza y actividades que se realizan para bien de la comunidad, la 
atención de los usuarios de los EBAIS en las clínicas periféricas, organización de actividades de los miembros de los EBAIS los jueves y viernes de $1 \mathrm{pm}$ a $3 \mathrm{pm}$, capacitación de los comités de salud, participación de la C.C.S.S. en la construcción y mantenimiento de los EBAIS. Estos temas emergentes podrían ser tema de otras investigaciones para tratarlos a profundidad.

\section{Triangulación de la información}

Para validar o dar confidencialidad al presente análisis se realizó una triangulación entre los resultados obtenidos de la opinión de los profesionales, los usuarios, los objetivos planteados para la investigación y las disposiciones de la O.P.S y la C.C.S.S en la Reforma del Sector Salud, las cuales también están contenidas en los módulos que se desarrollan en el curso especial de "Posgrado en gestión local de salud". Este análisis de realizó empleando las categorías mencionadas anteriormente.

\section{Aplicabilidad de los conocimientos}

La aplicabilidad surge de la revisión documental, la consulta a profesionales de la salud y a los usuarios, respecto a esta categoría se extrajeron aspectos significativos que el médico indicó son prioritarios a la aplicabilidad del módulo número tres, sobre la participación social en el campo de la salud. La participación social permite la redistribución del poder y en un incremento de la capacidad de los actores sociales para participar concertadamente en la comunidad.

El concepto de participación social definido en la C.C.S.S. (1997) se describe como:

Un proceso de interacción, negociación y concertación, que se establece, entre la población, la C.C.S.S. y las instituciones gubernamentales y no gubernamentales mediante acciones de organización, contribución y toma de decisiones, en los que prevalecen los derechos y deberes de los participantes y los intereses de todos, para la construcción de la salud en Costa Rica.
Durante la investigación este aspecto se puso en evidencia con la participación de usuarios en los comités de salud para la toma de decisiones que contribuyen al bienestar de la salud.

Los profesionales coinciden en que el curso les ayudó a adquirir destrezas y habilidades para trabajar con la comunidad y con el usuario, porque:

01MEF... "Con el curso me he aclarado lo que estoy haciendo, tengo seguridad en aplicar los conocimientos adquiridos, sobre todo en trabajo con la comunidad"...

Con esta opinión se evidencia que la capacitación recibida en el curso "Gestión Local de Salud" brinda seguridad en el quehacer de los médicos. Así como también se confirma con lo siguiente:

02MEG... "Ahora entiendo dónde encaja mi trabajo y me ha motivado bastante"...

03MET... "Entendí por qué se debe tener mayor comunicación con el usuario y los miembros del equipo"...

Uno de los contenidos del módulo tres sobre participación social da énfasis en la promoción, y participación en actividades de la comunidad, sobre ese aspecto la siguiente opinión de los profesionales es muy positiva:

04TS... "Lo que da mucho el curso "Gestión local de salud" son estrategias para la educación en salud y el uso de los sistemas de información, lo cual se utiliza día a día al atender al usuario, al trabajar con grupos de apoyo y con líderes de la comunidad”...

A pesar de que el contenido del curso se imparte a todos los profesionales en salud, contiene temas que están enfocados más para personal que labora a nivel local en especial en los EBAIS, como lo indica este informante:

05MC... "El curso de gestión local que realiza el CENDEISSS está enfocado para la atención de los EBAIS"...

La O.P.S. considera la promoción, la prevención, la curación y la rehabilitación 
de la salud como un proceso de construcción colectiva y participativa donde los actores principales son las comunidades y los agentes de salud.

Este otro aspecto se da en los EBAIS investigados, donde existe participación de la comunidad para la promoción y prevención de la salud, según la opinión de los informantes profesionales.

01MF ..."nosotros llegamos a las escuelas y a la comunidad durante una semana y brindamos lo que nos corresponde como entidad de la Caja.

01MF... "se realizan talleres de adultos mayores, adolescentes, campañas para todo público y se tiene bastante respuesta a la última campaña de papanicolao llegó muchísima gente”...

De las opiniones de los usuarios se puede inferir que en los EBAIS investigados, el usuario se siente seguro y es atendido con calor humano. El personal de salud que lo atiende lo respeta y le ayuda a resolver los problemas desde la familia. Le enseña a cuidar su salud y velar por el bienestar de su comunidad, por medio de actividades de promoción y participación social, como lo indican lo siguientes informantes:

UEF... "Este es un beneficio que más bien se puede decir, que no somos lo suficientemente agradecidos, porque yo me acuerdo que en vida de mi mamá como tenía yo que jalarla y a que costo, en cambio aquí nos ven, tenemos el consuelo de que hay medicina cerca aparte de que enriquece a la comunidad porque es bastante bonito"...

Sobre la importancia de la creación de los EBAIS

UEG... "Bueno, para nosotros la instalación del EBAIS ha sido una bendición para los vecinos"...

Y lo que más le interesa de la atención que se brinda en el EBAIS

UET... "Lo bonito es que el EBAIS lo envían a uno a hacerse exámenes al Hospital San Juan de Dios u otro"...
Con esta visión la comunidad tiene una función activa en los procesos de salud, en este caso se evidencia que existe una relación directa del médico del EBAIS con las comunidades por medio de los comités de salud, sin embargo las opiniones de los miembros de estos comités indican que se necesita mayor esfuerzo y apoyo de parte de las autoridades de salud y de los miembros de la comunidad para prevenir y controlar los problemas que se presentan, algunas opiniones al respecto son:

UCSG... " nos sentimos atados de manos"...

UCSG... "no hay acueductos de aguas negras y mucha gente descarga los tanques sépticos a las alcantarillas"...

Sobre el apoyo de los profesionales la actora indica,

UCSG... "la doctora colabora, el comité de salud también pero, el vecindario no"...

Sobre el apoyo de la comunidad

UCSG... "hay gente muy desconsiderada, tira todo a la calle... no les importa".

UCSG... "la gente no respeta, no considera, no cuida desde el hecho en que está ensuciando su propia comunidad"...

UCSG... "Hay mucho problema de drogadicción, hay Guardia Rural pero no hacen nada”.

Según Donabedian (1995). La capacidad social y cultural que tienen los sujetos de actuar plenamente en la solución y decisiones que se toman respecto a sus problemas cotidianos, políticos, sociales, no sólo distribuyen bienes materiales sino capitales y culturales que contribuyen a desarrollar capacidades de acción y decisión en los grupos beneficiarios.

Las diferentes opiniones refuerzan la responsabilidad del profesional de la salud a cargo de los EBAIS en realizar actividades de promoción y capacitación de sus 
miembros como de los actores sociales que funcionan como líderes de la comunidad.

UET... "El doctor a veces va al comunal a hacer exámenes a ver a todo el mundo, yo creo que ya va por dos o tres campañas que ha hecho"...

UEG... "El médico nos conoce y sabe a qué venimos, nos dedica tiempo, ve el expediente... nos escucha. Aunque sea allá en la escuela siempre me escucha y atiende"...

El Ministerio de Salud y la C.C.S.S. definen seis ambientes de desempeño de los EBAIS que son: el familiar, la comunidad, los establecimientos públicos y privados, el educativo, el laboral y el establecimiento de salud. Como se puede observar todas las funciones definidas anteriormente requieren amplios conocimientos y la aplicación de destrezas; así como la transformación de la actitud de los ejecutores que son el personal de salud, y la comunidad local. Para el cumplimiento de estas nuevas funciones es de vital importancia capacitar al personal de salud, quien a su vez educará a la comunidad y generará la participación comunal en la promoción de la salud. Este aspecto reafirma la importancia de las acciones del CENDEISSS en la realización del curso "Gestión local de salud" para profesionales que laboran en los EBAIS.

Los objetivos, estrategias didácticas de los módulos del curso especial de "Posgrado en gestión local de salud" proporcionan a los profesionales en salud, las herramientas necesarias para brindar atención integral, se evidencia que el enfoque que se da en los contenidos es para personal profesional de la salud que labora en el primer nivel de atención en los EBAIS, por lo que algunos contenidos no son de total aplicabilidad para los profesionales que laboran en clínicas periféricas u hospitales como es el caso del microbiólogo y el farmacéutico.

Según lo anterior, se afirma que existe aplicabilidad de los conocimientos recibidos en el curso especial de "Posgrado en gestión local de salud" en opinión de los profesionales y de los usuarios, coinciden ambos grupos en que la mayor aplicabilidad se da en los siguientes aspectos:

- Participación social en el campo de la salud por medio de las siguientes estrategias: visitas a las personas y grupos, participación en actividades realizadas como charlas a la comunidad, campañas de vacunación e interacción con actores sociales de la comunidad. Este aspecto coincide con el contenido del módulo 3.

- Atención integral por medio de las siguientes estrategias: abordar al usuario como un ser integral que piensa y actúa según su posición en la vida; identificar el EBAIS como equipo interdisciplinario; organizar la atención al usuario por grupos de edad con líneas de acción en diferentes escenarios, brindando atención individual, grupal y colectiva; y trabajo en equipo. Este aspecto coincide con el contenido del módulo 4.

- Análisis de la situación de salud, utilizando las estrategias de incorporación de actores sociales locales, fomentar la cultura de investigación en el personal de salud, analizar casos de estudio que interfieren en la salud de la población, interactuar con la comunidad, y equipo de apoyo. Este aspecto coincide con el contenido del módulo 6.

- Introducción al estudio de la calidad en los servicios de salud, identificando los elementos que caracterizan la calidad de atención en las actividades que se realizan para el mejoramiento de la calidad de atención. Este aspecto coincide con el contenido del módulo 10.

- $\quad$ La gestión clínica para el primer nivel de atención utilizando las estrategias 
como mantener una relación de equipo de salud con el usuario que permita aplicar los valores de la gestión clínica en la atención médico-usuario, visitas al hogar y utilización de referencia y contrarreferencia. Este aspecto coincide con el contenido del módulo 11.

Percepción sobre la atención brindada

La percepción surge de las opiniones de los consultados y para la triangulación se establece la relación con el análisis documental.

Los dos grupos de participantes consultados; profesionales y usuarios coinciden en que la atención que se brinda en los EBAIS es positiva al tomar en consideración los derechos determinantes del proceso de salud, enfermedad en los ámbitos biológico, económico, ecológico, y sociológico de las personas. (C.C.S.S. 1997). Sin embargo los aspectos percibidos como negativos por parte de los usuarios en relación con la atención recibida afectan en que ésta sea de calidad sobre todo en aspectos como: horario, cupo limitado para la atención, el tener que madrugar, hacer fila para obtener una ficha, trato menos humano en la clínica y hospital de referencia, problemas que afectan a toda la comunidad como son la contaminación ambiental, la basura, drogadicción, violencia intrafamiliar, ríos contaminados y otros problemas difíciles de resolver si no existe el apoyo de las instituciones gubernamentales y no gubernamentales.

Sobre este aspecto algunos usuarios informantes indicaron

UEF... "Usted tiene que madrugar como siempre como ir a la clínica, tiene que hacer fila, si quiere coger ficha 6:30 a.m. llega la secretaria le dice que ya no hay campo, porque sólo tantos pacientes atienen hoy, a veces es una pérdida de tiempo porque usted madruga, saca el rato, y resulta que cuando llegó tiene que devolverse"...

A veces no funciona madrugar para ser atendido porque,
UEF... "una vez fui como tres días seguidos y nunca tomé campo"..

Además se pasan por penalidades para llegar temprano y hacer fila ya que,

UET... "digamos uno que no tiene carro tiene que venirse en el primer bus a las cinco de la mañana, aún así no agarra campo, tiene que salir de la casa como a las cuatro de la mañana y caminar"...

Y además solo atienden un miembro por familia cada día, según el informante

ERG... "Yo al menos tengo un caso digamos común, mi señora está un poco engripada, yo estoy desde faltando diez para las cuatro aquí, para sacarle la cita a mi señora, pero yo también tengo unos exámenes para ver si me los pueden dar, pero ahí medio oí que no dan citas para dos de la misma familia, y la persona que está enfermita tiene que venir, hacer fila, para que le den la cita, y yo diría que si eso es cierto a mí no me parece"...

Según la documentación revisada en el módulo 10 del curso de capacitación se hace mención que la calidad es un producto de múltiples interrelaciones entre equidad, eficiencia, eficacia, la oportunidad de la atención y humanización de los servicios. Todos estos aspectos se toman en cuenta en la atención que se brinda a los usuarios en los EBAIS, pero no ha sido posible que se cumplan en su totalidad sobre todo por falta de recursos económicos, humanos y materiales. Todos estos aspectos están incluidos en las dimensiones a nivel personal, técnica y ambiental consideradas de importancia para definir si la atención que se brinda es de calidad. Como resultado de esta investigación se concluye que la atención que brindan los EBAIS es buena, pero deficiente en calidad.

No se podría brindar atención integral sin tomar en cuenta los actores sociales definidos como los miembros de la comunidad y los funcionarios que desarrollan todo su potencial individual y colectivo en pro de la salud. Según los resultados de la investigación las opiniones 
de los consultados reafirman que los EBAIS, dirigidos por el médico y equipo de apoyo permiten brindar una atención integral de la salud de las personas. Los equipos de apoyo realizan un trabajo coordinado y sistemático lo cual implica un amplio conocimiento actualizado del sector y del área de salud. Los aspectos positivos mencionados por los usuarios son: las actividades de promoción que se realizan en las comunidades como: ferias de salud, charlas en escuelas y colegios sobre temas de interés, campañas de vacunación, control del papanicolao, talleres con los adolescentes, atención personal y humanizada de parte del médico durante las consultas y existencia de los comités de salud. Las opiniones de los informantes son:

UEF... "tenemos el servicio de odontología”...

A pesar de la popularidad de que gozan las campañas de vacunación, se reconoce que

UEG... "nos dan charlas... hacen campañas de vacunación"...

La atención para las personas discapacitadas al nivel de EBAIS según la opinión de este usuario es,

UEG... "en el EBAIS dan trato especial a los discapacitados y adultos mayores"...

Los usuarios sin embargo indican que sienten un cambio en el trato que le dan en la clínica al recibir los servicios, comparado con el tipo de servicio que reciben en los EBAIS; califican la atención menos personal y menos humana, lo que interfiere en la calidad de ésta.

Algunos informantes indicaron

UEG..."Yo siento que en la clínica le cierran las puertas a los usuarios de los EBAIS con los ultrasonidos, como que nos les interesa, que es personal de otro lado, porque ya tiene que ser una situación muy grave para que a uno lo atiendan, aquí estamos a un nivel muy general, las especialidades son muy pocas también”...
Además se opina que las citas las dan con espacios muy largos,

UET... "mi referencia a otorrino... me cansé de ir y aquí estoy con mi oído enfermo, no hay especialistas en eso en la clínica, me dieron cita para el 2003”...

Sobre las citas para la realización de radiografías

UEF... "Tengo que realizarme una radiografía cada año y en la clínica no hay cupo, llego a la cita sin la radiografía"...

Sobre citas para la realización de otros estudios,

UET... "El médico del EBAIS me mandó a realizar un electrocardiograma y hace seis meses y no hay cupo"...

\section{Consideraciones finales}

Basándose en la información recopilada mediante entrevistas semiestructuradas, dirigidas a profesionales de la salud capacitados en "Gestión local de salud", grupos focales con usuarios que reciben atención en los EBAIS, análisis documental y la práctica laboral se concluye:

\section{Aplicabilidad de conocimientos según contenidos de los módulos, capacitación recibida $y$ práctica actual}

- $\quad$ El 100\% de los profesionales que recibieron el curso especial de posgrado en "Gestión local de salud" aplican total o parcialmente el contenido propuesto en los módulos 3, 4, 6, 10 y 11 . Esto se da en acciones de participación social en el campo de la salud en relación con promoción, capacitación organización de la comunidad, trabajo en actividades con la comunidad, visitas a personas y grupos e identificación de actores sociales, atención integral, abordando al usuario como 
un ser integral que piensa y actúa, identificar al EBAIS como equipo interdisciplinario, análisis del ASIS, identificación de los elementos que caracteriza la calidad en las actividades y la gestión clínica para el primer nivel de atención. Lo anterior permite indicar la importancia de estos módulos para la gestión local de la salud en concordancia con los diferentes servicios que deben brindar los EBAIS cumpliendo con los principios de la seguridad social de universalidad, solidaridad y equidad.

- $\quad$ Entre las actividades de participación social que se realizan con la comunidad, el profesional de salud que labora en EBAIS utiliza en primer lugar las ferias de salud, campañas de vacunación, toma de papanicolao, auto examen de mamas. Además, trabajo escolar por medio de charlas de educación sobre sexualidad, drogadicción y alimentación sana. Estas actividades permiten que el centro de atención deje de ser exclusivamente el individuo, para dirigirla a la familia, la comunidad y al ambiente, como lo indica la readecuación del modelo de la atención de la salud, el cual propicia un cambio de actitud de la sociedad con respecto a la salud. (Modernización C.C.S.S., 2002).

- La consulta para la atención del usuario en el EBAIS se organiza brindando atención de morbilidad en las mañanas, con cita restringida y en la tardes de lunes a miércoles se atienden los grupos prioritarios entre ellos crecimiento y desarrollo, prenatales, crónicos, atención del adulto mayor. Estas consultas se dan programadas con previa cita.

- $\quad$ La totalidad de profesionales entrevistados refieren que la población utiliza el $100 \%$ de los servicios que se brindan en la mañana, no así las consultas de la tarde en que se da ausentismo.

- Según las disposiciones de la C.C.S.S., la atención integral en salud al nivel de EBAIS se debe organizar por grupos de edad (niño, adolescente, atención de la mujer, adulto, adulto mayor) con líneas de acción en diferentes escenarios (familiar, educativo, laboral, comunitario, establecimientos de salud), brindando atención individual (educación para la salud, evaluación integral y participación en él. Sin embargo, la normativa que se emplea para organizar las consultas en los EBAIS investigados, interfiere en el cumplimiento de las políticas o lineamientos de la C.C.S.S. por lo que se debe reforzar la atención de morbilidad ampliando los cupos y los días de atención.

- Todos los EBAIS investigados cuentan con un comité de salud. Se concluye que éste es el apoyo más importante que tiene el EBAIS para la comunicación con la comunidad por lo que se debe reforzar la capacitación de sus integrantes y el apoyo por parte de todos los funcionarios de los EBAIS.

- Se brinda atención integral al usuario según la opinión del $100 \%$ de los usuarios entrevistados. Según el Ministerio de Salud, el equipo de salud es el responsable de brindar la atención integral en el primer nivel, entendida esta como un proceso de construcción social y por lo tanto debe tomar en consideración los derechos de la salud-enfermedad en los ámbitos biológicos, económico, ecológico y sociológico. 
- Todos los profesionales utilizan la referencia, sin embargo, son pocos los profesionales de la salud que aplican la contrarreferencia, en su mayoría la comunicación se da en forma verbal cuando es de interés para el médico conocer el seguimiento de sus pacientes.

- Todos los médicos que laboran en los EBAIS realizan entre 3 a 4 visitas domiciliares al mes de casos prioritarios, entre las frecuentes están las visitas a minusválidos, adultos mayores y las consideradas de urgencia. No se considera prioridad las visitas domiciliarias realizadas por el médico, por cuanto el tiempo disponible para esta actividad es limitado.

- La metodología para valorar la calidad en los servicios de salud brindados, es realizada solo parcialmente con el sistema del ciclo de mejoramiento continuo, la mayoría utiliza las encuestas de opinión y revisión general de los casos de estudio, según orden de importancia.

- $\quad$ En su totalidad los profesionales de salud afirman tener buena relación con los usuarios y equipos de trabajo. Se concluye que el puesto que tienen los profesionales en salud investigados entre ellos jefaturas de medicina, microbiología, trabajo social, nutricionista y enfermería les permite aplicar la mayoría de los conocimientos adquiridos, en el curso "Gestió Local de Salud".

- $\quad$ Con la investigación realizada se logró evidenciar la repercusión positiva del curso sobre todo en el desarrollo de estrategias para la atención integral de la salud, con la participación de la comunidad y el equipo básico. La deficiencia encontrada se da en que la calidad en la atención brindada es baja, por aspectos mencionados en relación con los pocos recursos para combatir los problemas sociales que afectan a las comunidades como son la drogadicción, violencia intrafamiliar, contaminación de los ríos, uso inadecuado de la basura, y pobreza en general.

\section{Percepción del usuario en relación con la atención recibida}

- Se concluye que los usuarios se sienten satisfechos con la creación de los EBAIS cerca de su comunidad. Sin embargo, se sienten insatisfechos con la consulta de morbilidad, para la cual deben hacer grandes filas y madrugar sin la seguridad de ser atendidos, debido a que los cupos son limitados. Los usuarios del EBAIS de Fátima y Guatuso no están satisfechos con la atención que se brinda en la consulta de odontología, por ser de cupo restringido, con una atención apenas básica, con trato poco cordial por parte del profesional a cargo.

- En su mayoría, los usuarios concluyen que la atención que se les brinda en la Clínica Dr. Marcial Fallas en los servicios de laboratorio, farmacia, especialidades y rayos x es menos personal y menos humana que la recibida en el EBAIS.

- Los usuarios perciben la participación del profesional de salud en la comunidad como buena, indicando que en su mayoría el médico se mantiene dentro del EBAIS, brindando consulta y es poco lo que sale a las comunidades. Los comités de salud son considerados para los usuarios y por los funcionarios de estos centros como la mano derecha para el EBAIS.

- Existe pasividad en la mayoría de 
las comunidades investigadas que se resisten a participar en la solución de sus propios problemas cuando se trata de brindar ayuda personal. Sin embargo, la mayoría acuden a las actividades de promoción de la salud que organiza los EBAIS en las comunidades como son: ferias de salud, campañas de vacunación, control de papanicolao, charla de auto cuidado, auto examen de mamas y alimentación adecuada.

- Los usuarios perciben que el profesional de la salud que está a cargo del EBAIS, cuenta con el conocimiento necesario para brindar atención integral, sin embargo el poco recurso y el local pequeño, limita la calidad de la atención que reciben por cuanto no se cumplen a cabalidad los principios de equidad, universalidad y oportunidad como se plantea en las políticas de salud.

- Los usuarios perciben que la calidad de atención que reciben es baja por las siguientes razones:

- La prestación de los servicios en su mayoría tiene una demanda de consulta superior a la esperada, lo que desfavorece la atención oportuna y puntual.

- La comunidad tiene una participación pobre en la solución de los problemas de salud que les afectan, ya que son comunidades con poco recurso para la resolución de sus problemas.

- Las comunidades presentan problemas difíciles de resolver, como drogadicción, violencia intra familiar, contaminación de los ríos, entre otros.
- La comunidad, en su mayoría, sigue exigiendo atención de morbilidad como atención prioritaria, resistiéndose a tener una participación activa en la solución de los problemas.

\section{Recomendaciones finales}

Con base en los hallazgos significativos se considera pertinente ofrecer algunas recomendaciones a las instancias responsables e involucradas en este estudio.

\section{A las instituciones involucradas con la atención de la salud. (C.C.S.S., MS)}

La ubicación de un EBAIS cerca de las comunidades no es la solución a todos los problemas de la salud, se necesita el apoyo de toda la comunidad incluyendo a las instituciones gubernamentales y no gubernamentales, por lo que se deben aplicar estrategias de motivación por parte de los médicos a cargo de los EBAIS para que las comunidades apoyen las acciones que se llevan a cabo en los EBAIS para el bienestar de la salud.

\section{Al CENDEISSS}

Realizar seguimiento y evaluación a los participantes que reciben el curso especial de posgrado en "Gestión local de salud" en las diferentes regiones del país, que permita hacer una comparación con los resultados de esta investigación o lo planificado por el CENDEISSS.

Incorporar a todos los miembros del equipo básico de atención integral de salud entre ellos los auxiliares de enfermería y ATAPS en la capacitación en "Gestión local de salud" sobre todo en los temas de participación social, atención integral, análisis de la situación de salud y calidad de la atención. 
Incluir como requisito dentro del curriculum de los profesionales de salud que laboran en los EBAIS el curso de "Gestión local de salud", lo que permitiría asegurar la capacitación del profesional de salud en los contenidos de los módulos $3,4,6,10$ y 11 que les brindarán las estrategias didácticas necesaria para el desempeño de su labor.

Ejecutar una evaluación curricular y readecuación de los programas de estudio del curso "Gestión local de salud" cada dos años de manera que éstos correspondan al contexto de la realidad social, política, económica, cultural y sobre todo a las necesidades de la sociedad costarricense.

Valorar las disposiciones de la C.C.S.S. sobre la abertura de EBAIS que no cuentan con el recurso necesario para la atención del usuario en forma integral, en especial lo referente al local. En su mayoría, los EBAIS en estudio son pequeños, con el inconveniente de que algunos comparten local, y personal como es el caso de Patarrá y Guatuso, Guido Uno, Tres y San Lorenzo.

\section{Recomendaciones para los profesionales capacitados en Gestión local de salud que trabajan en los EBAIS.}

Retomar con las jefaturas de salud, en reuniones, la importancia del uso de la referencia y contrarreferencia, por ser elemento básico para el control y seguimiento de la salud de los usuarios.

Se debe valorar la posibilidad de programar más visitas domiciliarias por mes para casos de seguimiento.

Los profesionales de la salud deberían realizar una evaluación al sistema empleado para el análisis de estudio de casos, considerando la posibilidad de retomar la guía de mejoramiento de la calidad desarrollada en el módulo 10.

Poner en práctica estrategias de motivación a las comunidades, para que todos sus miembros se involucren activamente en la solución de los problemas que les afectan.

Brindar apoyo oportuno a los comités de salud para la detección y solución de los problemas.

Realizar un análisis involucrando a la comunidad, sobre la posibilidad de resolver el problema de la consulta de morbilidad de manera tal que los usuarios reciban la atención, sin tener que madrugar para hacer filas, y con la seguridad de ser atendidos.

Realizar seguimiento y evaluación periódica sobre percepción del usuario en relación con la atención recibida, empleando procedimientos que favorezcan la apertura de espacios para recomendaciones y sugerencias.

Compartir los conocimientos recibidos en el curso "Especial de posgrado en Gestión local de salud" con los demás miembros de los equipos y comités de salud por medio de reuniones, charlas y entrega de documentación para análisis y sugerencias.

\section{Recomendaciones a los comités de salud}

Solicitar un programa de capacitación al EBAIS al iniciar su labor con seguimiento y evaluación periódicos por parte de éste.

Es importante que el comité de salud de Guatuso mantenga comunicación con el EBAIS por medio de un fax o teléfono, que les permita recibir orientación oportuna y eficaz para la labor que realizan en la comunidad.

Hacer un registro de recursos con que cuenta la comunidad, y comprometer a todos sus miembros en actividades propias de promoción y solución de problemas como es el caso de las Municipalidades, Cruz Roja, Guardia Rural, Iglesias, otras instituciones o grupos organizados. 


\section{Referencias bibliográfica}

Aguilar, H. Gestión de los servicios de enfermería. Caja Costarricense de Seguro Social. Centro de Desarrollo Estratégico e Información en Salud y Seguridad Social. Consultoría Visión Estratégica (CVE), San José, Costa Rica: EDNASSS, 2000.

Caja Costarricense de Seguro Social. "Propuesta participación social en salud". San José: Dirección Técnica de Servicios de Salud, 1993.

Caja Costarricense de Seguro Social. "Proyecto de Reforma del sector salud". Resumen ejecutivo. San José, Costa Rica: C.C.S.S. 1997.
Caja Costarricense de Seguro Social. Centro de Desarrollo Estratégico e Información en Salud y Seguridad Social. Curso especial en Gestión local de salud. San José, Costa Rica: C.C.S.S. 1999.

Donabedian, A. "Calidad de atención a la salud, continuidad y cambio en la búsqueda de la calidad". Revista ITAES, No. 2. Junio. C.C.S.S. 1995.

Hernández, R. y otros. Metodología de la investigación. México DF: McGraw Hill, 1991.

O.P.S, Sistema Locales de Salud. "La participación social”. Washington, DC. 1990.

Teresita Flores Jiménez Coordinadora Nacional del curso especial de "Posgrado de Gestión Local de Salud" CENDEISSS 